\title{
Profilaxis Antibiótica en Estomatología
}

\author{
Antibiotic Prophylaxis on Estomatology \\ Miguel Rodriguez Alfaro', Jonny Burga Sánchez², Victor Chumpitaz Cerrate², Roberto Varas Hilario², \\ Jaime Guerra Sanguinetti ${ }^{3}$, Roger López Bellido ${ }^{4}$, Juan Zegarra Cuya ${ }^{5}$
}

\begin{abstract}
La profilaxis antibiótica en cirugía consiste en utilizar un antibiótico de manera preventiva que sea activo frente a las bacterias que con mayor frecuencia causan infecciones posteriores a los procedimientos quirürgicos, y en mantener concentraciones tisulares eficaces durante toda la intervención quirúrgica y el periodo posterior donde se puede producir una bacteremia. Para establecer este régimen terapéutico es necesario tener un conocimiento apropiado de la flora bacteriana residente en boca y aquella flora patógena que con frecuencia infecta las heridas quirúrgicas. Para ello debemos hacer una racional elección del antibiótico a emplear así como utilizarlo durante el tiempo adecuado. Por lo general este tiempo no excede a las 24 horas de administración y en los esquemas profilácticos se emplean antibióticos como los betalactámicos, azálidos, lincosamidas y aminoglucósidos los cuales deberán ser escogidos dependiendo de la condición médica del paciente y de aspectos relacionados propiamente con la técnica quirúrgica ejecutada.
\end{abstract}

Palabras Clave: Profilaxis antibiótica, endocarditis, bacteremia, herida quirúrgica.

Surgical antibiotic prophylaxis consists in the use of an antimicrobial drug in a preventive way, that must be active against microorganisms that in high frequency causes posterior infections of our surgical wounds and maintain effective tissue concentrations along the surgery procedure and the posterior time when appears the bacteremia. To reach a successful treatment is necessary to have the knowledge of the resident bacterial flora and the pathogenous flora that infects our surgical wounds. We must choose the correct antibiotic and use it by the correct time. Generally, that time does not exceed 24 hours and in the prophylactic schemes are used some antimicrobial drugs like beta-lactams, azalides lyncosamides and aminoglycosides, that must be selected depending of the medical condition of the patient and some topic related directly with the surgical technique
employed.

Key Words: Chemoprophylaxis, endocarditis, bacteremia, surgical wound.

\section{INTRODUCCIÓN}

En muchas ocasiones nos enfrentamos a la atención de pacientes que presentan cuadros fisiopatológicos de fondo, los cuales requieren tratamiento odontológico principalmente quirúrgico, por lo cual resulta obligatoria la administración de antibióticos con la finalidad de evitar la infección del sitio quirúrgico o de otros focos a distancia. Es por esto, que frente a esta situación clínica, presentamos la información necesaria para la resolución de este punto que en ciertos aspectos resulta controversial o levanta muchas dudas por parte del profesional odontólogo.

La presentación de esta revisión busca facilitarle al profesional la elección de un determinado esquema, resaltando que es responsabilidad de cada cirujano - dentista conocer a plenitud las indicaciones, contraindicaciones, ventajas y desventajas de las diferentes alternativas a aplicar en cada paciente en particular.

\footnotetext{
' Responsable de la cátedra de Farmacología de la Facultad de Odontología UNMSM

${ }^{2}$ Catedráticos de Farmacología de la Facultad de Odontología UNMSM

${ }^{3}$ Cirujano Dentista de la Facultad de Odontología UNMSM

+ Bachiller de la Facultad de Odontología UNMSM

${ }^{5}$ Interno de la Facultad de Odontología UNMSM
}

\section{GeNERALIDADES}

En 1961, Burke demostró lo oportuno de administrar antibióticos para disminuir las infecciones de las heridas quirúrgicas en animales, hecho que fue confirmado en la práctica y desde entonces la antibioticoterapia profiláctica perioperatoria ha jugado un papel creciente en el campo de la cirugía. La profilaxis antibiótica en cirugía consiste en utilizar un antibiótico activo frente a las bacterias que con mayor frecuencia causan infecciones en la intervención en cuestión, y en mantener concentraciones tisulares eficaces durante toda la intervención quirúrgica. Su objetivo es minimizar las consecuencias de la colonización bacteriana, evitando la proliferación de las bacterias causantes de complicaciones infecciosas post-operatorias. Se pueden aislar bacterias patógenas en un alto porcentaje de las heridas quirúrgicas, en el momento de cerrarlas. Son poco numerosas pero pueden proliferar ya que encuentran en la herida un medio favorable para su desarrollo.

La profilaxis antibiótica se debe plantear en las tres situaciones clínicas siguientes:

1.- Cuando una complicación es frecuente, pero no fatal.

2.- Cuando es rara pero tiene una tasa de mortalidad elevada. 
3.- Cuando suele estar implicado un único tipo de microorganismo.

Los índices de infección de la herida quirúrgica son generalmente paralelos a la presencia de uno o más de los tres factores de riesgo clave:

a.- Condición médica general del paciente.

b.-Duración prolongada de la cirugía.

c.- Tipo de intervención quirúrgica (Clasificación de las intervenciones quirúrgicas de Altemeier).

En la práctica, la eficacia de la profilaxis se complica por diversos factores:

1.- A menudo suelen estar implicados diversos microorganismos.

2.- Estos microorganismos presentan una virulencia variable.

3.- Los microorganismos pueden originarse en distintos lugares.

4.- Pueden presentar una sensibilidad variable a los antibióticos administrados.

5.- Pueden aparecer bacteremias fisiológicas aleatorias.

6.- No hay estudios controlados que demuestren la eficacia de la profilaxis antibiótica.

Es útil agrupar a los procedimientos quirúrgicos de acuerdo a la frecuencia de infección post - operatoria. Para ello, tendremos en cuenta la Clasificación de las Intervenciones Quirúrgicas de Altemeier:

A.- Limpias: Su tasa de infección es de 1-5\% sin profilaxis. Se reduce a menos del $1 \%$ con la aplicación de profilaxis. Los procedimientos limpios son definidos como heridas operatorias no traumáticas ni infectadas sin inflamación aguda ni interrupción en la técnica aséptica. Se considera además herida limpia la que resulta de cirugía electiva sin ingresar a cavidades que normalmente se encuentran colonizadas como el tracto orofaríngeo. La profilaxis antibiótica no está indicada a menos que haya habido implantación de un cuerpo extraño como prótesis ortopédicas o válvulas cardíacas protésicas.

B.- Limpias - Contaminadas: La tasa de infección fluctúa entre el 5 y $15 \%$. se reduce a menos del $7 \%$ con profilaxis antibiótica. Resultan de la apertura controlada de cavidades contaminadas, mínima interrupción de la técnica aséptica, cirugía no electiva que en caso contrario sería considerada limpia y reintervención sobre incisión limpia en los 7 días siguientes.

C.- Contaminada: La tasa de infección es mayor al $15 \%$ y se reduce a menos del $15 \%$ con la aplicación de profilaxis. Resulta de inflamación aguda no purulenta, interrupción importante en la técnica aséptica, traumatismo de menos de 4 horas de evolución o heridas crónicas abiertas para injerto.
D.- Sucia: Su tasa de infección sin uso de antibiótico es mayor al $30 \%$. Con uso de antibiótico la tasa de infección disminuye. Resultan de perforación de cavidades contaminadas y traumatismo de más de 4 horas de evolución.

Las heridas LIMPIAS - CONTAMINADAS y CONTAMINADAS requieren obligatoriamente profilaxis antibiótica. Las heridas SUCIAS requieren tratamiento antibiótico completo. Ninguna herida producida en la cavidad oral será considerada como LIMPIA, por la colonización polimicrobiana de ésta.

\section{«Los antibióticos nunca sustituyen un buen juicio $y$ técnica quirúrgica»}

\section{Aspectos Microbiológicos de la Cavidad Oral}

Los microorganismos patógenos más usuales en cirugías limpias - contaminadas de cabeza y cuello con incisión de la cavidad oral o faringe son:

- Estafilococos aureus

- Estreptococos

- Anaerobios orales

- Bacterias coliformes

Los microorganismos más frecuentemente encontrados en cavidad oral como parte de la flora indígena con gran incidencia de contaminación de las heridas quirúrgicas se presentan en la tabla 1.

\section{Indicaciones para la Instauración de Esquemas de Profilaxis Antibiótica según la Condición Médica General del Paciente}

- Pacientes con condiciones cardíacas que impliquen riesgo de desarrollar endocarditis bacteriana. (ver tabla 2)

- Pacientes que utilizan prótesis articulares: Según la American Academy of Orthopedic Surgeons (AAOS), existe evidencia aunque limitada que justifica la utilización de profilaxis antibiótica en pacientes con prótesis articulares para prevenir la infección de estas, vía hematógena.

- Pacientes con problemas de inmunidad: En pacientes que se encuentran con un estado de inmunidad comprometido, las bacteremias producidas por procedimientos dentales invasivos pueden resultar peligrosas. En este grupo se pueden considerar pacientes col lupus eritematoso sistémico, neutropénicos, pacientes bajo terapia con corticoides, pacientes post transplante de órganos y otros. 


\section{Esquemas de Profilaxis Antibiotica para el Paciente Odontologico}

La administración del antimicrobiano debe realizarse simpre dentro de un periodo de 2 horas antes que se inicie el procedimiento quirúrgico. La duración óptima de la profilaxis perioperatoria. Con objeto de limitar la presión de selección de bacterias multiresistentes, la duración debe ser la más corta posible. Una única dosis preoperatoria suele ser suficiente, también puede considerarse una prevención limitada al período operatorio, pero en general, la duración no debe exceder las 24 horas. (En el caso de la endocarditis bacteriana la AHA recomienda que el periodo profiláctico no sea extendido por más de 6 a 8 horas).

Los antibioticos usados para profilaxis en cirugia pueden ser administrados vía endovenosa, intramuscular, oral e incluso tópica. La elección depende del tipo de cirugía; pero para la mayoría de procedimientos la vía endovenosa es la vía ideal, confiable y las concentraciones séricas del antibiótico son predecibles.

TABLA 1:

Microorganismos que conforman la flora indígena de la cavidad oral.

\begin{tabular}{|ll|}
\hline TIPO DE MICROORGANISMO & PRESENCIA \\
\hline GRAM POSITIVOS & \\
COCOS FACULTATIVOS & \\
Alfa Estreptococo & ++++ \\
Beta Estreptococo & + \\
Estreptococo No Hemolitico & +++ \\
Estafilococo & +++ \\
COCOS ANAEROBIOS & +++ \\
BACILOS FACULTATIVOS & \\
Actinomicetos & +++ \\
Lactobacilos & +++ \\
Difteroides & +++ \\
GRAMNEGATIVOS & \\
COCOS FACULTATIVOS & +++ \\
COCOS ANAEROBIOS & ++++ \\
BACILOS FACULTATIVOS & + \\
BACILOS ANAEROBIOS & \\
Bacteroides spp. & +++ \\
Prevotella, Porphyromonas spp. & +++ \\
Fusobacterium spp. & +++ \\
ESPIROQUETAS & +++ \\
LEVADURAS & +++ \\
VIRUS & $? ? ?$ \\
\hline
\end{tabular}

\section{Conclusiones}

1. La profilaxis antibiótica debe cubrir a los microorganismos que tienen más probabilidad de causar la infección de la herida operatoria.

2. Los antibióticos utilizados en los esquemas de profilaxis antibiótica deben ser bactericidas en las dosis utilizadas.

3. El empleo de dosis única como terapia profiláctica antibiótica brinda resultados similares al esquema de 2 dosis o de 24 horas.

4. La profilaxis antibiótica no deberá exceder en su administración a las 24 horas.

5. La farmacocinética difiere en los diferentes antibióticos, dependiendo de las vías de administración utilizadas.

Tabla 2

Condiciones cardiacas asociadas con endocarditis según la American Heart Association (AHA)

\section{CONDICIONES EN QUE SE RECOMIENDA} PROFILAXIS

\section{Categoría de alto riesgo}

Prótesis valvular cardíacaEpisodio previo de endocarditis infecciosaCardiopatías congénitas cianótica complejas (ej. Transposición de grandes vasos, tetralogía de Fallot, atresia tricuspídea)Derivación quirúrgica arterial sistémica-pulmonar (Blalock-Taussig)

\section{Categoría de riesgo moderado}

Otras cardiopatías congénitasCardiopatía reumática o alguna otra cardiopatía adquiridaCardiomiopatía hipertróficaPVM con insuficiencia mitral o soplo

\section{CONDICIONES EN QUE NO SE REQUIERE PROFILAXIS}

\section{Categoría de bajo riesgo}

Comunicación auricular no complicada tipo osteum secundum

Cierre quirúrgico de comunicaciones interauriculares e interventriculares

Ligadura de conducto arterioso persistente ( 6 meses posterior a la cirugía)

Cirugía de revascularización coronaria

PVM sin insuficiencia mitral ni soplo

Soplos fisiológicos, funcionales o sin peligroAntecedentes de enfermedad de Kawasaki sin disfunción valvular

Antecedentes de fiebre reumática sin disfunción valvular Marcapasos cardíacos (intravasculares y epicardíacos) 
Miguel Rodríguez Alfaro, Jonny Burga Sánchez, Victor Chumpitaz Cerrate, Roberto Varas Hilario, Jaime Guerra Sanguinetti, Roger López Bellido, Juan Zegarra Cuya

Tabla 3:

Regímenes profilácticos recomendados en cirugía maxilofacial

\begin{tabular}{|c|c|c|}
\hline SITUACIÓN & AGENTE & REGIMEN \\
\hline $\begin{array}{l}\text { Profilaxis } \\
\text { General } \\
\text { Estándar }\end{array}$ & Amoxicilina & $\begin{array}{l}\text { Adultos: } 2 \mathrm{~g} \mathrm{PO} \\
1 \text { hora antes del } \\
\text { procedimiento. } \\
\text { Niños: } 50 \mathrm{mg} / \mathrm{kg} \\
\text { PO } \\
1 \text { hora antes }\end{array}$ \\
\hline \multirow[t]{2}{*}{$\begin{array}{l}\text { Imposibilidad } \\
\text { vía oral }\end{array}$} & Cefazolina & $\begin{array}{l}\text { Adultos: } 1-2 \mathrm{gI} \\
\text { M-EV. } \\
\text { Niños: } 25 \mathrm{mg} / \mathrm{kg} \\
\text { IM - EV. } \\
30-15 \text { minutos } \\
\text { antes del } \\
\text { procedimiento. }\end{array}$ \\
\hline & Clindamicina* & $\begin{array}{l}\text { Adultos: } 600 \mathrm{mg} \\
\text { Niños: } 20 \mathrm{mg} / \mathrm{kg} \\
\text { PO.1 hora antes } \\
\text { del procedimiento } \\
\text { IM - EV. } \\
30-15 \\
\text { minutos antes del } \\
\text { procedimiento }\end{array}$ \\
\hline \multirow{2}{*}{$\begin{array}{l}\text { Alergia a } \\
\text { Penicilina }\end{array}$} & $\begin{array}{l}\text { Cefalexina o } \\
\text { Cefadroxilo** }\end{array}$ & $\begin{array}{l}\text { Adultos: } 2 \mathrm{~g} \text { PO. } \\
\text { Niños: } 50 \mathrm{mg} / \mathrm{kg} \\
\text { PO. } 1 \text { hora antes } \\
\text { del procedimiento. }\end{array}$ \\
\hline & $\begin{array}{l}\text { Azitromicina o } \\
\text { Claritromicina }\end{array}$ & $\begin{array}{l}\text { Adultos: } 500 \mathrm{mg} \\
\text { PO } \\
\text { Niños: } 15 \mathrm{mg} / \mathrm{kg} \\
\text { PO I hora antes } \\
\text { del procedimiento }\end{array}$ \\
\hline \multicolumn{3}{|c|}{$\begin{array}{l}\text { * Alternativamente asociado con Gentamicina } 1.7 \mathrm{mg} / \mathrm{kg} \text { EV (Adultos) } \\
12.5 \mathrm{mg} / \mathrm{Kg} \text { EV (Niños), asociación que ha demostrado ser eficaz en } \\
\text { cirugía de cabeza y cuello que incluyen procedimientos en mucosa } \\
\text { orofaríngea. } \\
\text { ** Riesgo reacción cruzada alérgica entre cefalosporinas y penicilinas } \\
\text { del } 5 \text { al } 10 \%\end{array}$} \\
\hline
\end{tabular}

\section{REFERENCIAS BIBLIOGRÁFICAS}

1. Strachan I. Antibiotecoterapia profiláctica perioperatoria. Acta Médica 1998; 8 (1): 105-9.

2. Little J., Falace D., Miller C. Tratamiento odontológico del paciente bajo tratamiento médico. 1998.

3. Scholar E., Pratt W. The Antimicrobial Drugs. $2^{\text {nd }}$ edition. Oxford University Press. 2000

4. Rutkauskas J. Infectious Disease Clinics of North America. Oral Infection. 1999.

5. Montazem A. Antibiotic Prophylaxis in Dentistry. October November 1998. Number 5 \& 6 volume 65:388-392.

6. Jacobs M.R. Optimisation of antimicrobial therapy using pharmacokinetic and pharmacodynamic parameters. Clin Microbiol Infect 2001; 7:589 - 596 .

7. Goodman \& Gilman: Las Bases Farmacológicas de la terapéutica. Décima edición. México. McGraw Hill interamericana. 2003.
8. Mandell, Douglas and Bennett. Principles and practice of infectious diseases. Fourth edition. New York. Churchill Livingtone.1995.

9. Fine D., Hammond B., Loesche W., Clinical use of antibiotics in dental practice. International Journal of antimicrobial agents 9, $235-238,1998$

10. Diaz L., Mares G., Conceptos actuales sobre profilaxia antibiotica para endocarditis bacteriana en odontología.

Revista ADM, Vol LVI, N ${ }^{\circ}$, enero - febrero , pp 32 - 38 . 1999.

11. Lindeboom, J., Baas E., Kroon F., Prophylactic single dose administration of $600 \mathrm{mg}$ clindamycin versus 4-time administration of $600 \mathrm{mg}$ clindamycin in orthognatic surgery: A prospective randomized study in bilateral mandibular sagittal ramus osteotomies. Oral surgery, oral medicine, oral pathology Vol $95, \mathrm{~N}^{\circ} 2$ february 2003 